\title{
The LIG Multi-Criteria System for Video Retrieval
}

\author{
Stéphane Ayache \\ Laboratoire d'Informatique \\ Fondamentale de Marseille
}

\author{
Georges Quénot, Laurent Besacier \\ Laboratoire d'Informatique de Grenoble \\ Georges.Quenot@imag.fr
}

\begin{abstract}
The LIG search system uses a user-controlled combination of six criteria: keywords, phonetic string, similarity to example images, semantic categories, similarity to already identified positive images, and temporal closeness to already identified positive images.

Categories and Subject Descriptors: H.3.3 [Information Storage and Retrieval]: Information Search and Retrieval

General Terms: Algorithms, Experimentation.

Keywords: Video retrieval, multi-criteria search.

\section{THE LIG VIDEO RETRIEVAL SYSTEM}

The LIG search system [1] uses a user-controlled combination of six criteria: keywords, phonetic string (new in 2009), similarity to example images, semantic categories, similarity to already identified positive images, and temporal closeness to already identified positive images (Figure 1). The system outputs an ordered list of relevant shots for each topic after interaction with the user (initial query and multiple relevance feedback). This system was used for TRECVID [2], VideOlympics [3] and the Star Challenge [4].
\end{abstract}

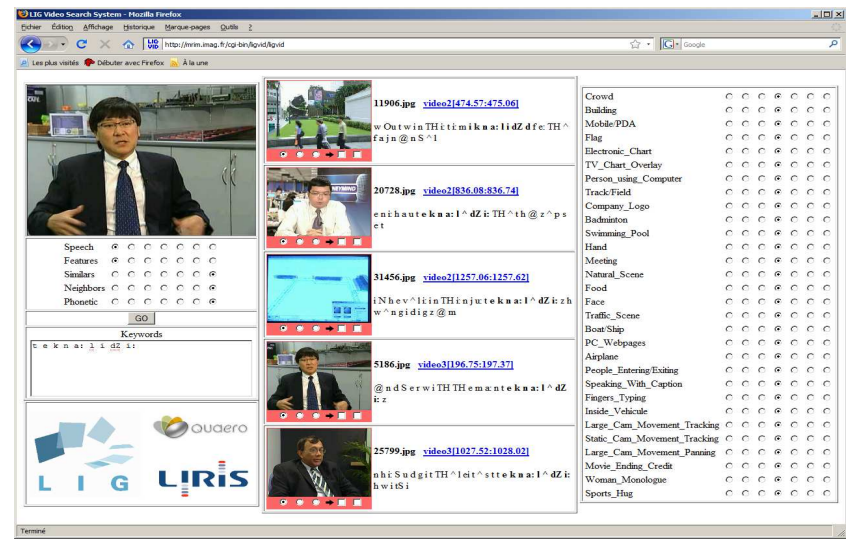

Figure 1: The LIG video retrieval system

Criterion 1: Keyword based search. The keyword based search is done using a vector space model. The words present in the ASR-MT transcription are used as vector

Copyright is held by the author/owner(s). CIVR'09, July 8-10, 2009 Santorini, GR ACM 978-1-60558-480-5/09/07. space dimensions. Stemming ans stopword list are used. Relevance is first assigned to speech segments and then projected onto overlapping shots.

Criterion 2: Phonetic string based search. Video documents are transcribed at the phonetic level using a subset of the International Phonetic Alphabet (IPA). Queries are made in the same way and the search is done using a phonetic string spotting algorithm.

Criterion 3: Similarity to image examples. Visual similarity between key frames and image examples is looked for using color and texture characteristics $(4 \times 4 \times 4$ color histograms and $8 \times 5$ Gabor transforms). normalized and the turned into a relevance value for each feature.

Criterion 4: Feature based search. Video shots are ranked according to the 36 pre-classified categories used in the TRECVID 2007 high level feature detection task.

Criteria 5 and 6: Visual similarity and temporal closeness. Visual similarity and temporal closeness (within the video stream) to already retrieved images can be used for the search. These images have to be marked by the user as positive examples for similarity based search and/or as positive examples for temporal closeness based search (relevance feedback).

Combination of search criteria. The user can dynamically define his search strategy according to the topic and/or the looking of the retrieved images. Each search criterion can be independently configured and given a global weight for the search. The per-criterion relevances are linearly combined according to the criteria weights to produce the final key frame relevance.

\section{REFERENCES}

[1] Stéphane Ayache and Georges Quénot. CLIPS-LSR Experiments at TRECVID 2006. In Proceedings of the TRECVID 2006, 13-14 November 2006.

[2] Alan F. Smeaton, Paul Over, and Wessel Kraaij. Evaluation campaigns and TRECVid. In MIR '06: Proceedings of the 8th ACM International Workshop on Multimedia Information Retrieval, pages 321-330, New York, NY, USA, 2006. ACM Press.

[3] Cees G. M. Snoek, Marcel Worring, Ork de Rooij, Koen E. A. van de Sande, Rong Yan, and Alexander G. Hauptmann. VideOlympics: Real-time evaluation of multimedia retrieval systems. IEEE MultiMedia, 15(1):86-91, January-March 2008.

[4] http://hlt.i2r.a-star.edu.sg/starchallenge. 